\title{
A numerical approach for pressure transient analysis of a vertical well with complex fractures
}

\author{
Yizhao Wan ${ }^{1} \cdot$ Yuewu Liu' ${ }^{1}$. Wenchao Liu ${ }^{1} \cdot$ Guofeng Han $^{1} \cdot$ Congcong Niu$^{1}$
}

Received: 30 July 2015 / Revised: 14 December 2015 / Accepted: 11 April 2016 / Published online: 25 May 2016

(C) The Chinese Society of Theoretical and Applied Mechanics; Institute of Mechanics, Chinese Academy of Sciences and Springer-Verlag Berlin Heidelberg 2016

\begin{abstract}
A new well test model for a vertical fractured well is developed based on a discrete-fracture model in which the fractures are discretized as one dimensional (1-D) entities. The model overcomes the weakness of complex meshing, a large number of grids, and instability in conventional stripe-fracture models. Then, the discrete-fracture model is implemented using a hybrid element finite-element method. Triangular elements are used for matrix and line elements for the fractures. The finite element formulation is validated by comparing with the semi-analytical solution of a single vertical fractured well. The accuracy of the approach is shown through several examples with different fracture apertures, fracture conductivity, and fracture amount. Results from the discrete-fracture model agree reasonably well with the stripefracture model and the analytic solutions. The advantages of the discrete-fracture model are presented in mesh generation, computational improvement, and abilities to handle complex fractures like wedge-shaped fractures and fractures with branches. Analytical results show that the number of grids in the discrete-fracture model is $10 \%$ less than stripefracture model, and computational efficiency increases by about $50 \%$. The more fractures there are, the more the computational efficiency increases.
\end{abstract}

Keywords Fractured well - Discrete-fracture model . Numerical well test . Hybrid finite element method . Complex fracture

Yuewu Liu

liuyuewulxs@126.com

1 Institute of Mechanics, Chinese Academy of Sciences, Beijing 100190, China

\section{Introduction}

As an important technology in the development of a low permeability reservoir, artificial fracturing technology has been widely used in oil fields, especially the development of unconventional gas like shale gas, coal-bed methane and tight gas, etc. There is no natural productivity in unconventional gas after drilling because of the complex geological structure, dense reservoir, and low abundance of natural gas. This kind of low permeability needs artificial fracturing to obtain industrial oil and gas flow. Fractures generated by fracturing extend into the formation and provides the flow channel for oil and gas. Complex fractures around the wellbore have a significant effect on the fluid flow in formation. The analysis of the flow in porous media can be used to estimate the fracturing measures, so as to guide development of the shale gas, coal-bed methane, and tight gas.

Since the 1960s, significant progress has been made towards understanding and modeling of flow processed in a fractured well. In 1972, Gringarten et al. [1] established a model of infinitely conducting fractures and uniform flux fractures with a hypothetical zero-radius well. Later, CincoLey et al. [2] extended the Greens functions approach of Gringarten et al. to consider the more realistic cases of finite conductivity vertical fractures. The concept of fracture description developed by Gringarten, Ramey, and CincoLey was applied in many other models with fractures like multi-fractured horizontal wells [3], partially penetrating fractures [4], and so on.

The analytical or semi-analytical approach solutions can be obtained only if the system under consideration is simple. Analytical solutions consider: (1) the reservoir is homogeneous, (2) the fracture has uniform aperture and conductivity, and (3) the well is crossed by only one fracture. The aim of 
the numerical method [5-10] is to conveniently overcome the limitations of the analytic methods. However, current numerical methods meet another problem, which is the two different length scales when it overcomes the limitations of analytic methods. Usually, the fracture is presented by a rectangle and discretized as the same as a matrix [11], which is called the stripe-fracture model. The large number of grids is required even if the aperture of the fracture is the smallest mesh scale. Another problem caused by the scale difference is the instability and divergence [12]. Although the equivalent seepage resistance method [13], which enlarges the aperture and reduces the permeability under the conditions of the same fracture flux and can be used, it is limited by the formation coefficient, fracture permeability and many other factors. The equivalent seepage resistance method can cause errors and not be used in complex fracture cases.

The discrete-fracture method is a new type of technology to describe fracture. In this method, fractures are discretized as one dimensional (1-D) entities, and the flow in the fractures is only along the direction of fractures, so the number of grids decreases and the instability and divergence are eliminated. Furthermore, Noorishad and Mehran [14] were among the early authors to use 1-D entities to represent fractures. Then, many authors used this method for the reservoir simulation from a single phase flow to a two-phase flow [15-19]. No work can be found in the literature applying the discretefracture model to a well test. Many authors [20,21] validate the discrete-fracture model by comparing the recovery curves and water saturation contours with the stripe-fracture model. However, the sensitivity of these variables is low. It has to be examined whether the discrete-fracture model can be used in well tests when the pressure derivative is so sensitive.

In this paper, a numerical well test model based on the discrete-fracture method is developed. The discrete-fracture model is implemented using a hybrid element finite-element method. Triangular elements are used for matrix and line elements for the fractures. The finite element formulation is validated by comparing with the semi-analytical solution of a single vertical fractured well. The accuracy of the approach is shown through several examples with different fracture apertures, fracture conductivity, and fracture amount. The advantages of the discrete-fracture model are presented in mesh generation, computational improvement, and ability to handle complex fractures like wedge-shaped fractures and fractures with branches.

\section{Statement of the problem}

The transient pressure behavior for a fractured well can be studied by analyzing the solution of the differential equa-

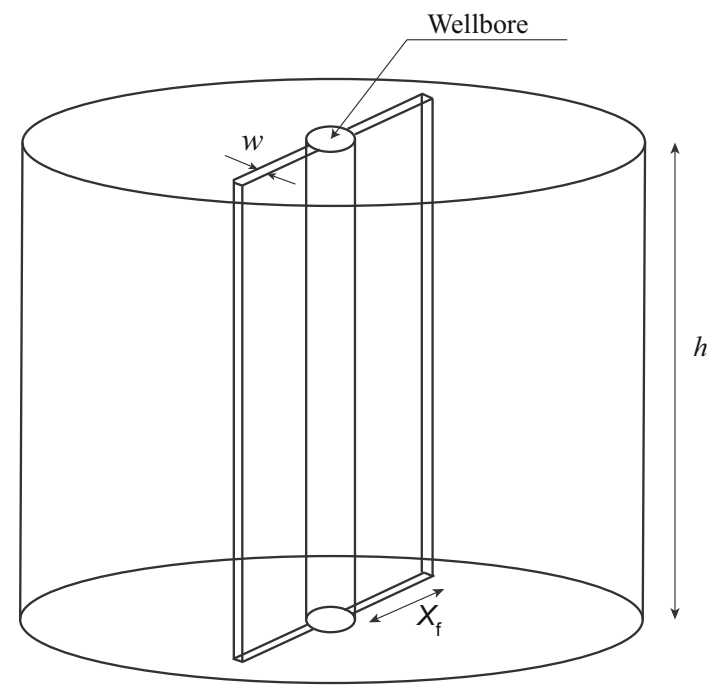

Fig. 1 Finite conductivity vertical fracture model

tions that describe this phenomenon with proper initial and boundary conditions. To simplify the derivation of models, the following assumptions are made. (1) An isotropic, homogeneous, horizontal, slab reservoir is bounded by upper and lower impermeable strata. The reservoir has uniform thickness, $h$, permeability, $K_{\mathrm{m}}$, and porosity, $\phi_{\mathrm{m}}$, which are independent of pressure. (2) The formation is produced through a vertically fractured well. The wellbore is intersected by a fully penetrating vertical fracture of permeability, $K_{\mathrm{f}}$, porosity, $\phi_{\mathrm{f}}$, aperture, $w$, and half-length, $X_{\mathrm{f}}$. (3) The porous medium contains a slightly compressible fluid of viscosity, $\mu$ and compressibility $C_{\mathrm{t}}$. The system above defined is shown in Fig. 1.

\section{Mathematical model}

The main idea of the discrete-fracture model is a 1-D representation of the fractures. The governing equations are discretized in two dimensional (2-D) form for the matrix and in 1-D form for the fractures. The idea is examined in Fig. 2 . The whole domain is represented by $\Omega$, the matrix by $\Omega_{\mathrm{m}}$, and the fracture by $\Omega_{\mathrm{f}}$. a

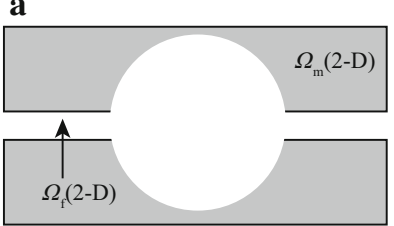

b

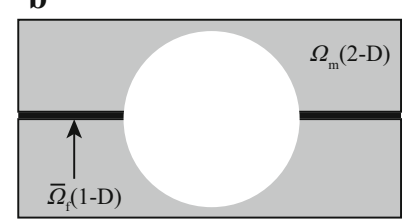

Fig. 2 Schematic representation of the discrete-fracture approximation. a Stripe-fracture model. b Discrete-fracture model 
Let $F E Q$ represent the flow equations. According to Fig. 2, the integral form of these equations for the stripfracture model can be written as

$$
\iint_{\Omega} F E Q \mathrm{~d} \Omega=\iint_{\Omega_{\mathrm{m}}} F E Q \mathrm{~d} \Omega_{\mathrm{m}}+\iint_{\Omega_{\mathrm{f}}} F E Q \mathrm{~d} \Omega_{\mathrm{f}} .
$$

The same integral for the discrete-fracture model is written as

$$
\iint_{\Omega} F E Q \mathrm{~d} \Omega=\iint_{\Omega_{\mathrm{m}}} F E Q \mathrm{~d} \Omega_{\mathrm{m}}+w \int_{\bar{\Omega}_{\mathrm{f}}} F E Q \mathrm{~d} \bar{\Omega}_{\mathrm{f}},
$$

where $\overline{\Omega_{\mathrm{f}}}$ represents the fracture part of the domain as a 1-D entity and $w$ represents the aperture of fractures.

In the discrete-fracture model, it assumes that inside the fracture, all variables remain constant in the lateral direction that is ignoring the pressure gradient of the vertical direction. The aperture $w$ of fracture appears as an explicit factor in front of the 1-D integral for the consistency of the integral form. The control equation of the flow in fractures can be simplified for the 1-D form.

The governing equations of this problem are obtained by combining Darcy's law and mass conservation based on the discrete-fracture model description. For the matrix, the transient equation of pressure can be written as

$\frac{K_{\mathrm{m}}}{\mu} \frac{\partial^{2} p_{\mathrm{m}}}{\partial x^{2}}+\frac{K_{\mathrm{m}}}{\mu} \frac{\partial^{2} p_{\mathrm{m}}}{\partial y^{2}}=\phi_{\mathrm{m}} C_{\mathrm{t}} \frac{\partial p_{\mathrm{m}}}{\partial t}$,

where $K_{\mathrm{m}}$ is the matrix permeability, $\mu$ is the fluid viscosity, $p_{\mathrm{m}}$ is matrix pore pressure, $\phi_{\mathrm{m}}$ is the matrix porosity, and $C_{\mathrm{t}}$ is the total compressibility.

For fractures, the transient equation of pressure based on the discrete-fracture model can be written as

$\frac{K_{\mathrm{f}}}{\mu} \frac{\partial^{2} p_{\mathrm{f}}}{\partial l^{2}}=\phi_{\mathrm{f}} C_{\mathrm{t}} \frac{\partial p_{\mathrm{f}}}{\partial t}$,

where $K_{\mathrm{f}}$ is the fracture permeability, $p_{\mathrm{f}}$ is the fracture pressure, $\phi_{\mathrm{f}}$ is the fracture porosity, and $l$ is the local coordinate of fractures, which is shown in Fig. 3.

$p_{\mathrm{m}}=p_{\mathrm{i}}, p_{\mathrm{f}}=p_{\mathrm{i}}$,
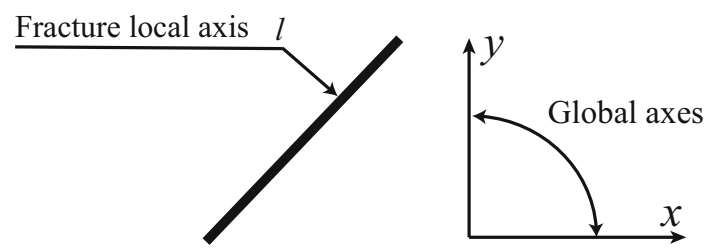

Fig. 3 Sketch of fracture local axis. Initial conditions for this problem are as follows where $p_{\mathrm{i}}$ is the initial formation pressure.

The constant production rate boundary conditions around the wellbore are as follows

$\left.\sum_{j=1}^{N} L_{j} h \frac{K}{\mu} \frac{\partial p_{j}}{\partial n}\right|_{\Gamma_{i}}=B q+C \frac{\mathrm{d} p_{\mathrm{w}}}{\mathrm{d} t}$,

$p_{j}=p_{\mathrm{i}}$,

where $L_{j}$ is the matrix element edge on the wellbore, $B$ is a fluid volume factor, $q$ is the production rate, $p_{\mathrm{w}}$ is the bottom hole pressure, and $N$ is the number of edges on the wellbore.

Constant pressure at the reservoir outer boundary is given by

$\left.p\right|_{\Gamma_{\mathrm{o}}}=p_{\mathrm{i}}$,

or no flow condition

$\left.\frac{\partial p}{\partial n}\right|_{\Gamma_{\mathrm{o}}}=0$.

\section{Numerical method}

The geometry is discretized by triangular elements for the matrix and line elements for the fractures, which is presented in Fig. 4. Firstly, the node and triangle are numbered just like normal mesh without fractures. Secondly, line elements for fractures are marked separately.

We use the Galerkin weighted residual method and finite-element discretization to solve Eqs. (3) and (4). The variational form of these equations can be written as

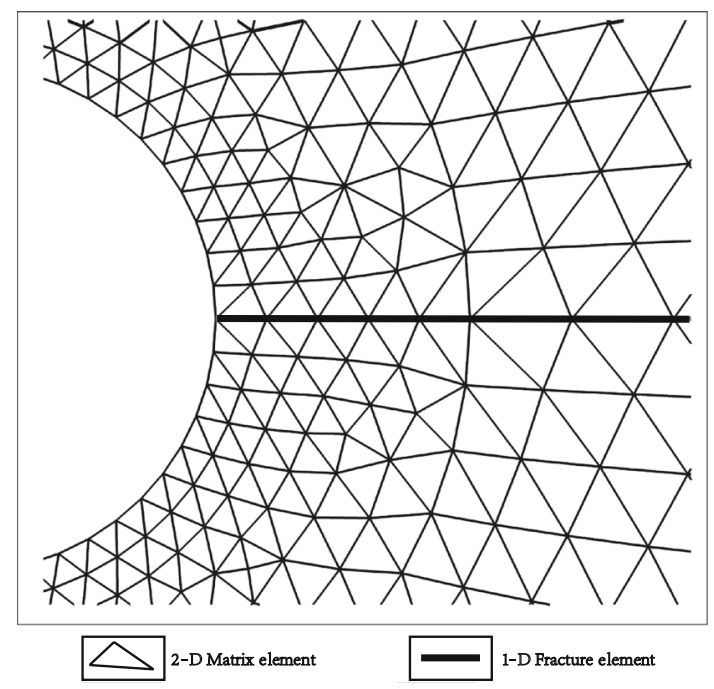

Fig. 4 Discretization of the discrete-fracture media 
$\iint_{A}\left(\frac{K_{\mathrm{m}}}{\mu} \frac{\partial^{2} p_{\mathrm{m}}^{\mathrm{e}}}{\partial x^{2}}+\frac{K_{\mathrm{m}}}{\mu} \frac{\partial^{2} p_{\mathrm{m}}^{\mathrm{e}}}{\partial y^{2}}-\phi_{\mathrm{m}} C_{\mathrm{t}} \frac{\partial p_{\mathrm{m}}^{\mathrm{e}}}{\partial t}\right) \delta p_{\mathrm{m}}^{\mathrm{e}} \mathrm{d} A=0$,

and

$\int_{l}\left(\frac{K_{\mathrm{f}}}{\mu} \frac{\partial^{2} p_{\mathrm{f}}^{\mathrm{e}}}{\partial l^{2}}-\phi_{\mathrm{f}} C_{\mathrm{t}} \frac{\partial p_{\mathrm{f}}^{\mathrm{e}}}{\partial t}\right) \delta p_{\mathrm{f}}^{\mathrm{e}} \mathrm{d} l=0$.

The weak form of these variational equations can be written as

$$
\begin{aligned}
& \iint_{A}\left(\frac{K_{\mathrm{m}}}{\mu} \frac{\partial p_{\mathrm{m}}^{\mathrm{e}}}{\partial x} \frac{\partial \delta p_{\mathrm{m}}^{\mathrm{e}}}{\partial x}+\frac{K_{\mathrm{m}}}{\mu} \frac{\partial p_{\mathrm{m}}^{\mathrm{e}}}{\partial y} \frac{\partial \delta p_{\mathrm{m}}^{\mathrm{e}}}{\partial y}+\phi_{\mathrm{m}} C_{\mathrm{t}} \frac{\partial p_{\mathrm{m}}^{\mathrm{e}}}{\partial t} \delta p_{\mathrm{m}}^{\mathrm{e}}\right) \mathrm{d} A \\
& \quad=\int_{S} \delta p_{\mathrm{m}}^{\mathrm{e}} \frac{K_{\mathrm{m}}}{\mu} \frac{\partial p_{\mathrm{m}}^{\mathrm{e}}}{\partial n} \mathrm{~d} S
\end{aligned}
$$

and

$$
\int_{l}\left(\frac{K_{\mathrm{f}}}{\mu} \frac{\partial p_{\mathrm{f}}^{\mathrm{e}}}{\partial l} \frac{\partial \delta p_{\mathrm{f}}^{\mathrm{e}}}{\partial l}+\phi_{\mathrm{f}} C_{\mathrm{t}} \frac{\partial p_{\mathrm{f}}^{\mathrm{e}}}{\partial t} \delta p_{\mathrm{f}}^{\mathrm{e}}\right) \mathrm{d} l=\left.\delta p_{\mathrm{f}}^{\mathrm{e}} \frac{K_{\mathrm{f}}}{\mu} \frac{\partial p_{\mathrm{f}}^{\mathrm{e}}}{\partial l}\right|_{1} ^{2} .
$$

Right terms of Eqs. (12) and (13) can be offset between the nearby elements by continuous conditions. The matrix element pressure is approximated as

$p_{\mathrm{m}}^{\mathrm{e}}=N_{\mathrm{m} i} p_{\mathrm{m} i}^{\mathrm{e}}+N_{\mathrm{m} j} p_{\mathrm{m} j}^{\mathrm{e}}+N_{\mathrm{m} k} p_{\mathrm{m} k}^{\mathrm{e}}$,

where $p_{\mathrm{m} i}^{\mathrm{e}}, p_{\mathrm{m} j}^{\mathrm{e}}, p_{\mathrm{m} k}^{\mathrm{e}}$ are the three node pressure, $N_{\mathrm{m}}$ is the element interpolating function, which is written as

$N_{\mathrm{m} i}=a_{i}+b_{i} x+c_{i} y$.

The time derivative terms are discretized by forward difference as follows

$\frac{\partial p_{\mathrm{m}}^{\mathrm{e}}}{\partial t}=\frac{p_{\mathrm{m}}^{\mathrm{e}, n+1}-p_{\mathrm{m}}^{\mathrm{e}, n}}{\Delta t}$.

Then, the matrix element equations are as follows

$$
\begin{gathered}
A\left(\frac{K_{\mathrm{m}}}{\mu} b_{i}^{2}+\frac{K_{\mathrm{m}}}{\mu} c_{i}^{2}+\frac{\phi_{\mathrm{m}} C_{\mathrm{t}}}{6 \Delta t}\right) p_{\mathrm{m} i}^{\mathrm{e}, n+1}+ \\
A\left(\frac{K_{\mathrm{m}}}{\mu} b_{i} b_{j}+\frac{K_{\mathrm{m}}}{\mu} c_{i} c_{j}+\frac{\phi_{\mathrm{m}} C_{\mathrm{t}}}{12 \Delta t}\right) p_{\mathrm{m} j}^{\mathrm{e}, n+1}+ \\
A\left(\frac{K_{\mathrm{m}}}{\mu} b_{i} b_{k}+\frac{K_{\mathrm{m}}}{\mu} c_{i} c_{k}+\frac{\phi_{\mathrm{m}} C_{\mathrm{t}}}{12 \Delta t}\right) p_{\mathrm{m} k}^{\mathrm{e}, n+1}- \\
\frac{L}{3} \frac{\partial p_{\mathrm{m} i}^{\mathrm{e}, n+1}}{\partial n}-\frac{L}{6} \frac{\partial p_{\mathrm{m}(j, k)}^{\mathrm{e}, n+1}}{\partial n}= \\
\frac{\phi_{\mathrm{m}} C_{\mathrm{t}}}{6 \Delta t} p_{\mathrm{m} i}^{\mathrm{e}, n}+\frac{\phi_{\mathrm{m}} C_{\mathrm{t}}}{12 \Delta t} p_{\mathrm{m} j}^{\mathrm{e}, n}+\frac{\phi_{\mathrm{m}} C_{\mathrm{t}}}{12 \Delta t} p_{\mathrm{m} k}^{\mathrm{e}, n},
\end{gathered}
$$

where $A=\left|\begin{array}{lll}1 & x_{i} & y_{i} \\ 1 & x_{j} & y_{j} \\ 1 & x_{k} & y_{k}\end{array}\right|, b_{i}=-\frac{1}{2 A}\left|\begin{array}{cc}1 & y_{j} \\ 1 & y_{k}\end{array}\right|, c_{i}=$ $\frac{1}{2 A}\left|\begin{array}{rr}1 & x_{j} \\ 1 & x_{k}\end{array}\right|(i, j, k)$, and $x_{i}, y_{i}, x_{j}, y_{j}, x_{k}, y_{k}$ are the triangle node coordinates.

The same operation is implemented for fracture line elements. The fracture pressure is approximated as

$p_{\mathrm{f}}^{\mathrm{e}}=N_{\mathrm{f} i} p_{\mathrm{f} i}^{\mathrm{e}}+N_{\mathrm{f} j} p_{\mathrm{f} j}^{\mathrm{e}}$,

where $p_{\mathrm{fi}}^{\mathrm{e}}, p_{\mathrm{f} j}^{\mathrm{e}}$ are the line element node pressure, $N_{\mathrm{f}}$ is the interpolating function for linear line element given by

$N_{\mathrm{f} i}=\frac{l_{j}-l}{L}, N_{\mathrm{f} j}=\frac{l-l_{i}}{L}$.

Then, the fracture element equations are as follows

$$
\begin{aligned}
& \left(\frac{K_{\mathrm{f}}}{\mu} \frac{1}{L}+\frac{\phi_{\mathrm{f}} C_{\mathrm{t}}}{3 \Delta t}\right) p_{\mathrm{f} i}^{\mathrm{e}, n+1}+\left(-\frac{K_{\mathrm{f}}}{\mu} \frac{1}{L}+\frac{\phi_{\mathrm{f}} C_{\mathrm{t}}}{3 \Delta t}\right) p_{\mathrm{f} j}^{\mathrm{e}, n+1} \\
& =\frac{\phi_{\mathrm{f}} C_{\mathrm{t}}}{3 \Delta t} p_{\mathrm{f} i}^{\mathrm{e}, n}+\frac{\phi_{\mathrm{f}} C_{\mathrm{t}}}{3 \Delta t} p_{\mathrm{f} j}^{\mathrm{e}, n}
\end{aligned}
$$

where $L=\left|l_{i}-l_{j}\right|$ is the line element length.

The wellbore boundary condition is given by

$\left.\sum_{j=1}^{N} L_{j} h \frac{K}{\mu} \frac{\partial p_{j}^{n+1}}{\partial n}\right|_{\Gamma_{\mathrm{i}}}+\frac{p_{\mathrm{w}}^{n+1}}{\Delta t}=B q+C \frac{p_{\mathrm{w}}^{n}}{\Delta t}$.

The fracture matrix system is superimposed on the matrix due to the original non-fracture formulation. This concept is illustrated in Fig. 5. The large-scale sparse linear equations are solved by the open source library SuperLU [22].

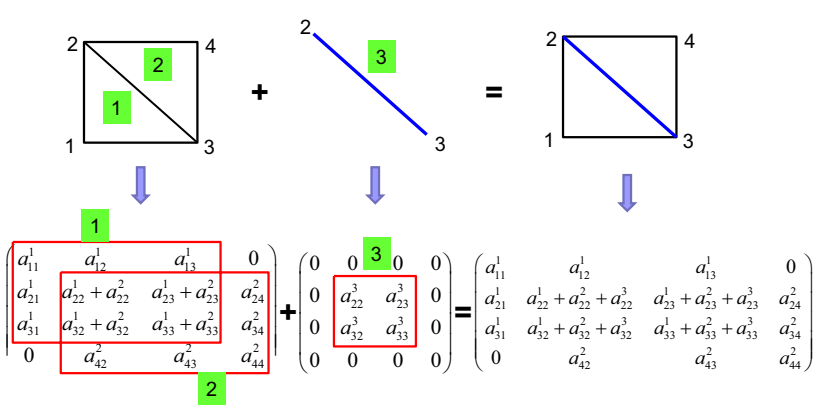

Fig. 5 Schematic illustrating combining matrix and fracture element equation 


\section{Results and discussion}

\subsection{Validation of the model}

\subsubsection{Single fracture case}

Firstly, we compare the solutions of this paper with CincoLey's semi-analytical solutions. The results of these simulations are presented in Fig. 6 in the form of a log-log plot of the dimensionless bottom hole pressure $p_{\mathrm{wD}}=$ $2 \pi K_{\mathrm{m}} /(q \mu B)\left(p_{\mathrm{i}}-p_{\mathrm{w}}\right)$ versus $t_{\mathrm{Df}}=K_{\mathrm{m}} t /\left(\phi_{\mathrm{m}} \mu C_{\mathrm{t}} X_{\mathrm{f}}^{2}\right)$ for different values of dimensionless fracture conductivity $F_{\mathrm{CD}}=\left(K_{\mathrm{f}} w\right) /\left(K_{\mathrm{m}} X_{\mathrm{f}}\right)$. In Fig. 6 solutions are presented for four values of $F_{\mathrm{CD}}: 0.2 \pi, \pi, 10 \pi, 100 \pi$. The solutions of the discrete-fracture model are in excellent agreement with the solutions of Cinco-Ley et al., which means the procedure for the discrete-fracture model is correct. Although, in the particular case of $F_{\mathrm{CD}}=0.2 \pi$, the discrete-fracture results differ from the semi-analytical solutions for $t_{\mathrm{Df}}<0.01$, this departure is due to the effect of the finite value of $r_{\mathrm{w}}$, which is used in the discrete-fracture model, but not in the semianalytical solutions.

The nature of the discrete-fracture model is to ignore the changes of variables in the lateral direction. Inside the fractures, the flow is one-dimensional. Therefore, this simplification would not cause a large error if the fracture aperture $w$ is small. The applicability in the case of large $w$ needs evaluation. Comparison between the solutions of the discretefracture model and the stripe-fracture model is proposed. We consider a single fracture with aperture of 0.001 and $0.05 \mathrm{~m}$ cross the well. Other reservoir conditions and parameters are: initial pressure is $50 \mathrm{MPa}$ with circle no-flow boundaries, the matrix permeability is $10 \times 10^{-3} \mu \mathrm{m}^{2}$; porosity is 0.1 , viscosity is $1 \mathrm{mPa} \cdot \mathrm{s}$, volume factor is $1 \mathrm{~m}^{3} / \mathrm{m}^{3}$, production rate is $50 \mathrm{~m}^{3} / \mathrm{d}$, wellbore storage factor is $0.001 \mathrm{~m}^{3} / \mathrm{MPa}$, and

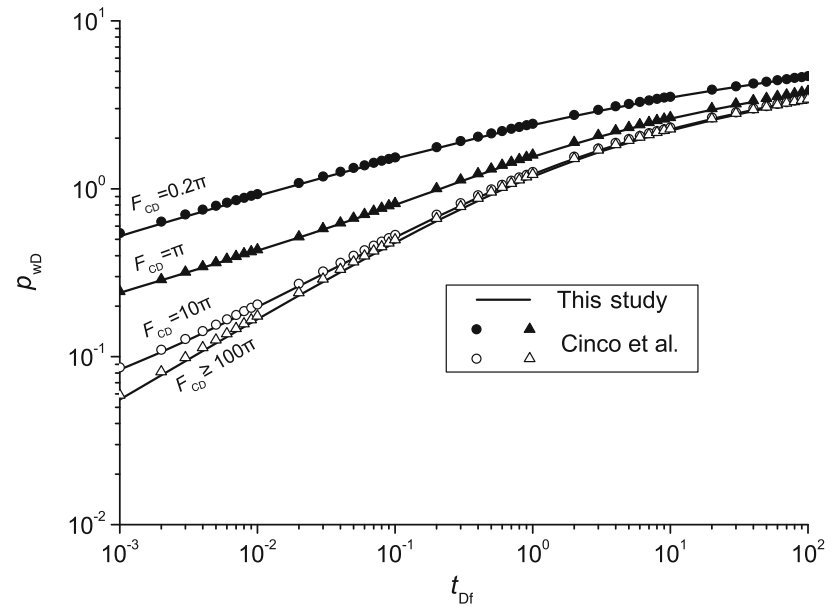

Fig. $6 \mathrm{Log}-\log$ plot of $p_{\mathrm{wD}}$ vs $t_{\mathrm{Df}}$ for various of $F_{\mathrm{CD}}$ : comparison of the discrete-fracture model and the semi-analytical results fracture half-length is $10 \mathrm{~m}$. The comparison of the two numerical methods is summarized in Fig. 7 for different $F_{\text {CD }}$.

Figure $7 \mathrm{a}$ and $\mathrm{c}$ is the $\log -\log$ plot of dimensionless bottom hole pressure $p_{\mathrm{wD}}$ and pressure derivative $p_{\mathrm{wD}}^{\prime}$ vs $t_{\mathrm{Df}}$ for various values of $w$. Figure $7 \mathrm{~b}$ and $\mathrm{d}$ is the pressure distribution along the fracture. As can be seen from Fig. 7, there is a very good agreement between the results of the discretefracture model and the stripe-fracture model, even though the fracture aperture is $0.05 \mathrm{~m}$ which is much larger than the value in reality.

Table 1 presents the maximum relative deviation of all the time points for different values of $F_{\mathrm{CD}}$. The largest relative deviation is less than $2 \%$. The relative deviation of the discrete-fracture model gets larger with the increase of fracture aperture and conductivity.

\subsubsection{Multi-fracture case}

In order to further verify the applicability of the discretefracture model, the cases of two, three, four, and six half rotational symmetry fractures are simulated by the discretefracture model and the stripe-fracture model. The fracture aperture is $0.001 \mathrm{~m}$ and other parameters are the same with the single fracture case. The meshes of the two models are presented in Fig. 8.

Results of the stripe-fracture model and the discretefracture model are shown in Fig. 9. There is also a good agreement between the discrete-fracture model and the stripe-fracture model in multi-fracture cases.

Comparison of pressure contours is presented in Fig. 10. As can be seen, the results of the discrete-fracture model are in excellent agreement with the stripe-fracture model. The tiny difference is in fractures. Contour lines of the discretefracture model are sharper because fractures in the discretefracture model have no geometry thickness.

From the above analysis, the results of the discretefracture model are in excellent agreement with the analytical solutions and numerical solutions of the stripe-fracture model for single and multiple fractures. This shows that the implementation of discrete-fracture model, the solution method, and the numerical algorithms employed are fundamentally sound.

\subsection{Evaluation of the model}

\subsubsection{Preprocessing}

Fractures in the discrete-fracture model are discretized as linear line elements. The fractures have no thickness in geometry, so there is no requirement to draw the rectangle for fracture just like the stripe-fracture model. Geometry modeling and mesh generation are easier especially for intersected 

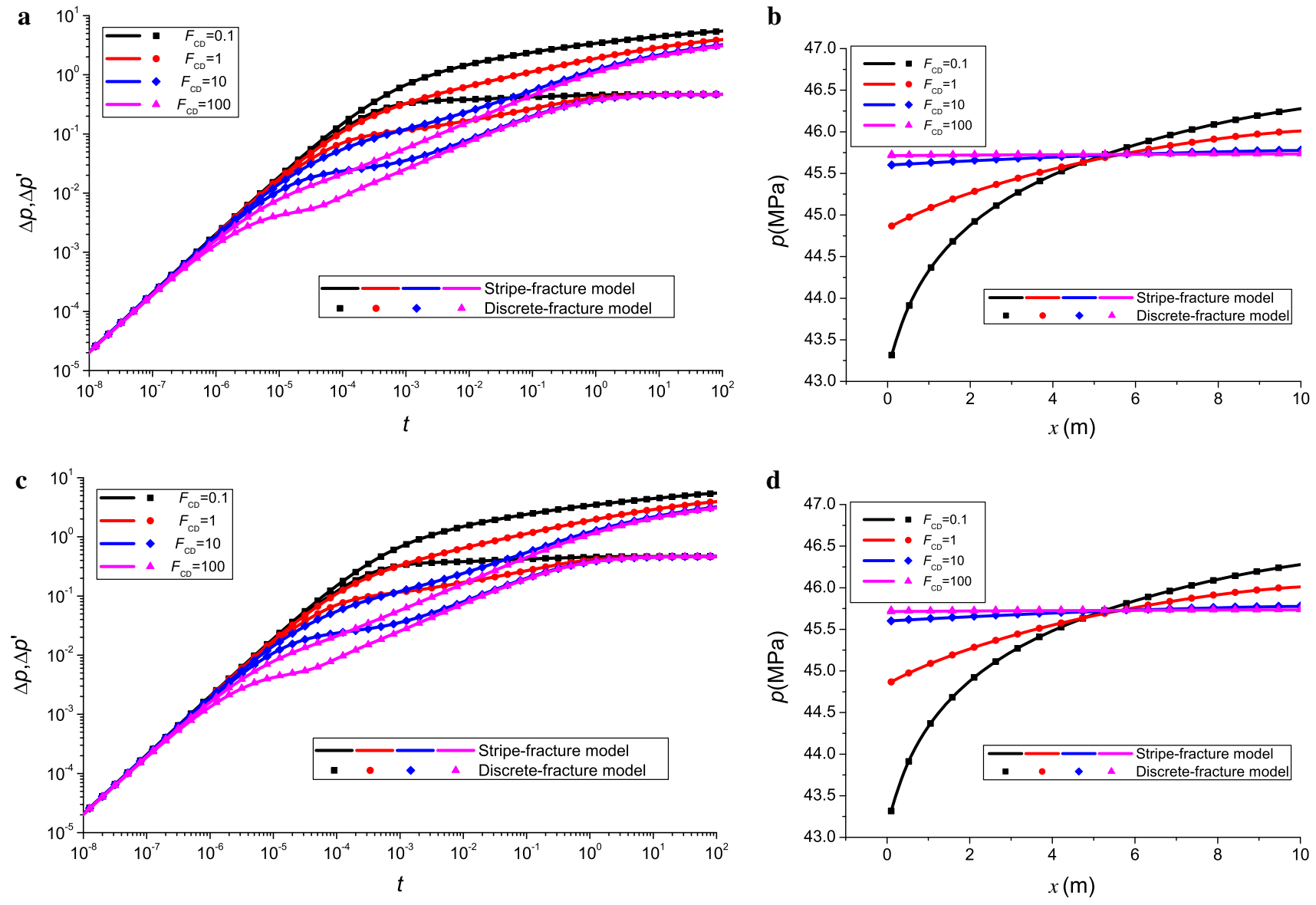

Fig. 7 (Color online) Comparisons between the results for the discrete-fracture model and the stripe-fracture model. a Log-log plot of $\Delta p$ and $\Delta p^{\prime}$ vs $t$ for $w=0.001 \mathrm{~m}$. b $p$ along fracture for $w=0.001 \mathrm{~m}$. $\mathbf{c} \log -\log$ plot of $\Delta p$ and $\Delta p^{\prime}$ vs $t$ for $w=0.05 \mathrm{~m}$. $\mathbf{d} p$ along fracture for $w=0.05 \mathrm{~m}$

Table 1 Relative deviation between the results from the discrete-fracture model and the stripe-fracture model

\begin{tabular}{lllll}
\hline$w(\mathrm{~m})$ & $F_{\mathrm{CD}}=0.1(\%)$ & $F_{\mathrm{CD}}=1(\%)$ & $F_{\mathrm{CD}}=10(\%)$ & $F_{\mathrm{CD}}=100(\%)$ \\
\hline 0.001 & 0.223 & 0.694 & 0.342 & 1.03 \\
0.05 & 0.226 & 1.020 & 0.712 & 1.65 \\
\hline
\end{tabular}

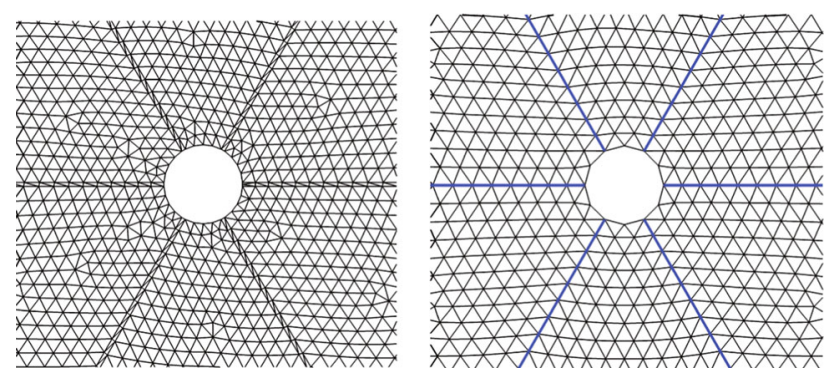

Fig. 8 Mesh of multiple-fracture case for the stripe-fracture model (left) and discrete-fracture (right) model

fractures case, which is presented in Fig. 11. The discretefracture model can also do simulation at various values of $w$ without regenerating meshes, because the aperture $w$ of the fracture appears as an input factor in the governing equations.

\subsubsection{Computational performance}

Computational efficiency is closely related to the number of mesh nodes. Compared to the stripe-fracture model, the number of mesh nodes in the discrete-fracture model is less because of 1-D discretization for fractures. Table 2 presents the number of meshes and the computational efficiency for the stripe-fracture model and the discrete-fracture model. As can be seen, the number of mesh nodes in the discrete-fracture model can be reduced by $10 \%-20 \%$, and the computational efficiency can be increased by $50 \%$. The improvement will enhance with the increase of fracture number. 


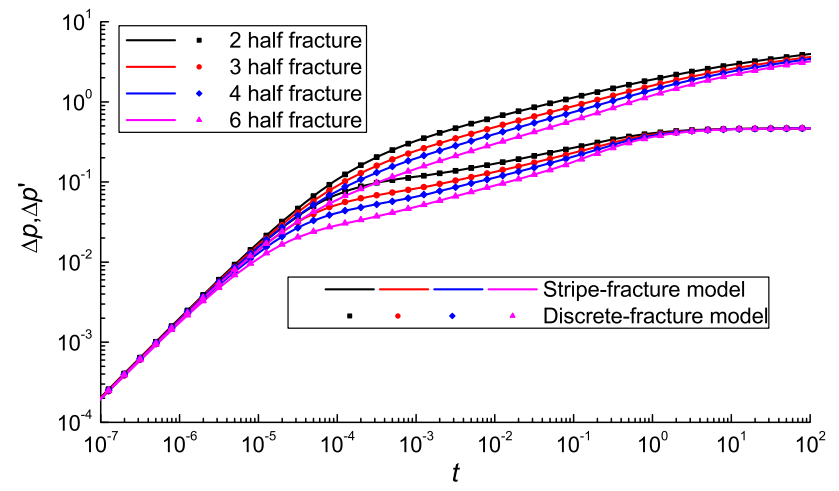

Fig. 9 (Color online) Log- $\log$ plot of $\Delta p$ and $\Delta p^{\prime}$ vs $t$ for various numbers of fractures

\subsubsection{Complex fractures}

(1) Non-uniform aperture

It is customarily assumed that the fractures have uniform aperture. However, it is conceivable that fracture aperture changes with the distance from the wellbore. $w$ is the function of fracture position, which is written as

$w=f(l)$.
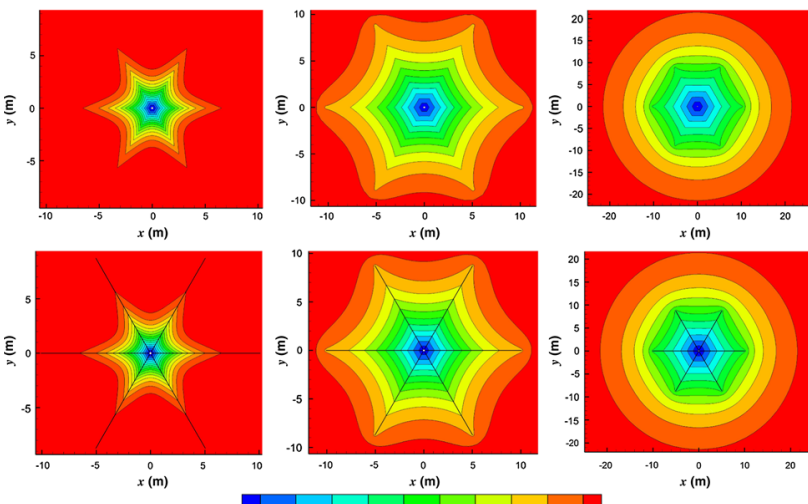

$p(\mathrm{MPa}) 46.5046 .8947 .28$ 47.67 48.06 48.44 48.8349 .2249 .6150 .00

Fig. 10 (Color online) Pressure contours for the stripe-fracture model (up) and the discrete-fracture model (down) at $t=0.01 \mathrm{~h}, t=0.1 \mathrm{~h}$, and $t=1 \mathrm{~h}$ (from left to right)
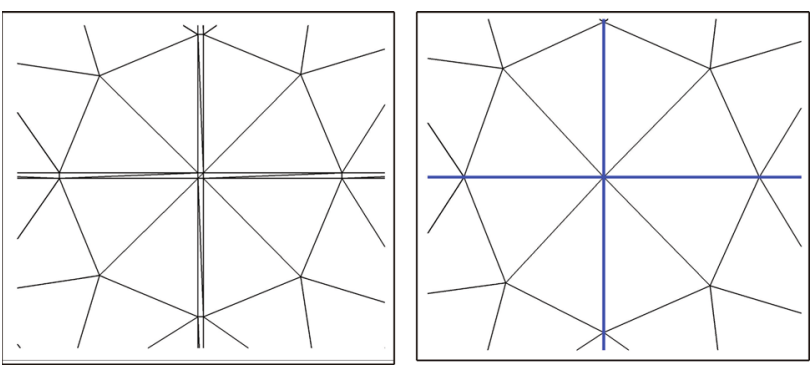

Fig. 11 Meshes of the stripe-fracture model (left) and the discretefracture model (right) for intersected fractures
A wedge-shaped fracture is the representation of nonuniform aperture fracture. It is presented in Fig. 12. The aperture of fracture in the end of the wellbore is $w_{0}$, and the half-length is $l_{0}$. The fracture aperture function is given by

$w=\frac{w_{0}}{l_{0}}\left(l_{0}-l\right)$

For this kind of complex fracture, the customary simulation method is to construct the geometry model according to the actual shape of fracture and generate meshes. It is complex and causes grid deformity. In contrast, there is no need to regenerate meshes for the discrete-fracture model. The simple way is to input the aperture functions $f(l)$ of the fractures.

We also compare the results of the wedge-shaped fractures from the discrete-fracture model and the stripe-fracture model. It is presented in Fig. 13. As can be seen from this figure, there is an excellent agreement between the results of the two kinds of models. The discrete-fracture model is more flexible and convenient for wedge-shaped fractures. For other shape fractures, it is just needed to set the specific form of Eq. (22). The advantages of the discrete-fracture model are more prominent for complex fractures. It can be applied in multi-fractured horizontal wells and many other situations.

\section{(2) Fractures with branches}

The position and angle of the fractures generated by the complicated process of hydraulic fracturing is randomly scattered. Another case of complex fractures is the one with branch fractures, which is presented in Fig. 14. Besides the bi-wing fractures directly connected with the wellbore, there are branch fractures connected with bi-wing fractures. As can be seen, branch fractures are mainly horizontal and vertical and some branch fractures are intersecting with each other. We assume that the conductivity of branch fractures is smaller than the bi-wing fractures.

The discrete-fracture model is very suitable for the simulation of this complicated situation due to the advantages presented in Sect. 5.2. The mesh of this case is presented in Fig. 15, which is very easy to generate because of the application of the discrete-fracture model.

Figure 16 shows the propaganda of pressure from 0.01 to $10 \mathrm{~h}$. As can be seen from this figure, pressure firstly spreads through the bi-wing fractures, which are directly connected with the wellbore. Then, pressure drop occurs in the branch fractures. The speed of pressure expansion in the bi-wing fractures is faster than in the branch fractures, which can be explained by the fact that the conductivity of bi-wing fractures is larger. When expanded to all the fractures, pressure propagates homogeneously in the formation.

Discrete-fracture model is a powerful tool to model the flow problem related to fractures like the multiple fractured 
Table 2 Relative deviation between the results from the discrete-fracture model and the stripe-fracture model

\begin{tabular}{|c|c|c|c|c|c|}
\hline Fracture number & Model & Node number & Node reduce percent $(\%)$ & computational time (s) & Efficiency increasing (\%) \\
\hline \multirow[t]{2}{*}{3} & Stripe-fracture & 23596 & 9.87 & 231 & 13.85 \\
\hline & Discrete-fracture & 21268 & & 199 & \\
\hline \multirow[t]{2}{*}{4} & Stripe-fracture & 28234 & 24.41 & 532 & 52.26 \\
\hline & Discrete-fracture & 21341 & & 254 & \\
\hline \multirow[t]{2}{*}{6} & Stripe-fracture & 29596 & 10.47 & 573 & 47.29 \\
\hline & Discrete-fracture & 26496 & & 302 & \\
\hline
\end{tabular}

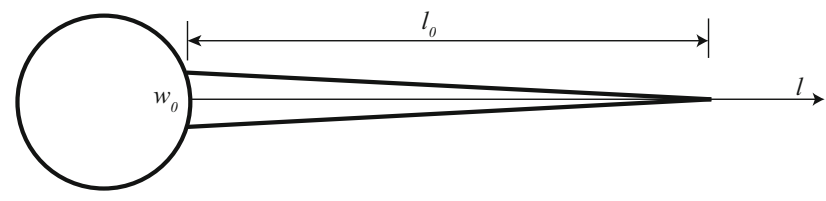

Fig. 12 Sketch of the wedge-shaped fracture

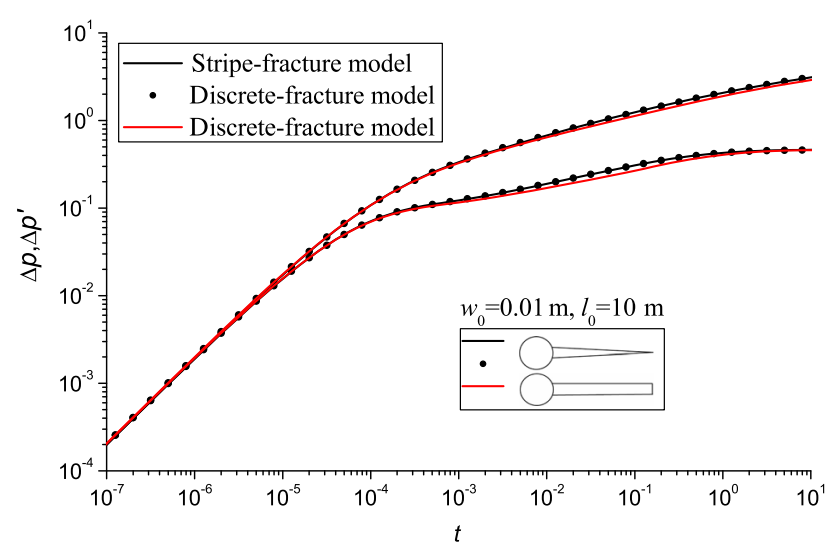

Fig. 13 (Color online) Log-log plot of $\Delta p$ and $\Delta p^{\prime}$ vs $t$ for wedgeshaped fractures

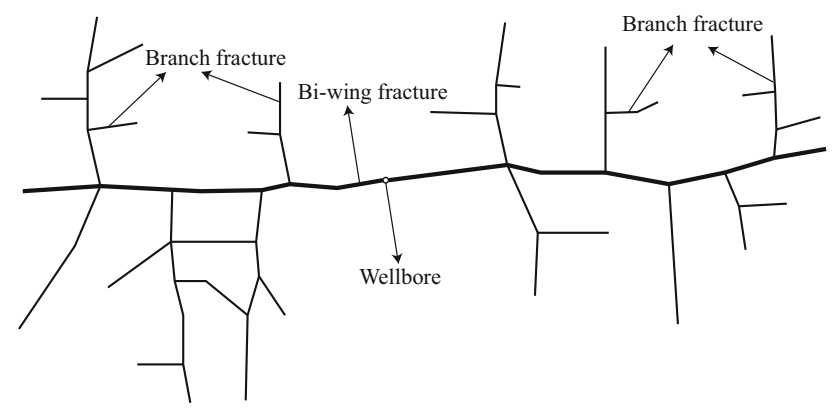

Fig. 14 The schematic diagram of fractures with branches

horizontal wells, which are the most important development for shale gas and tight gas. If the information of hydraulic fractures is obtained from logging or microseismic technology, an accurate simulation can be performed by the discrete-fracture model.

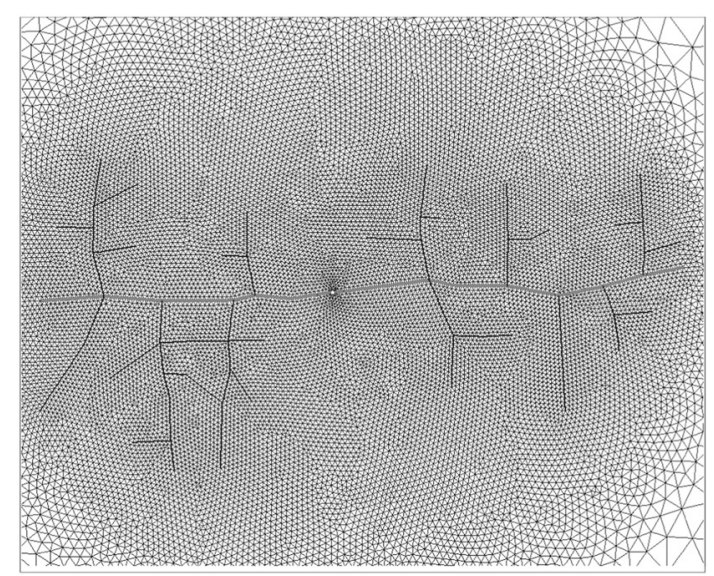

Fig. 15 Mesh in the case of fractures with branches
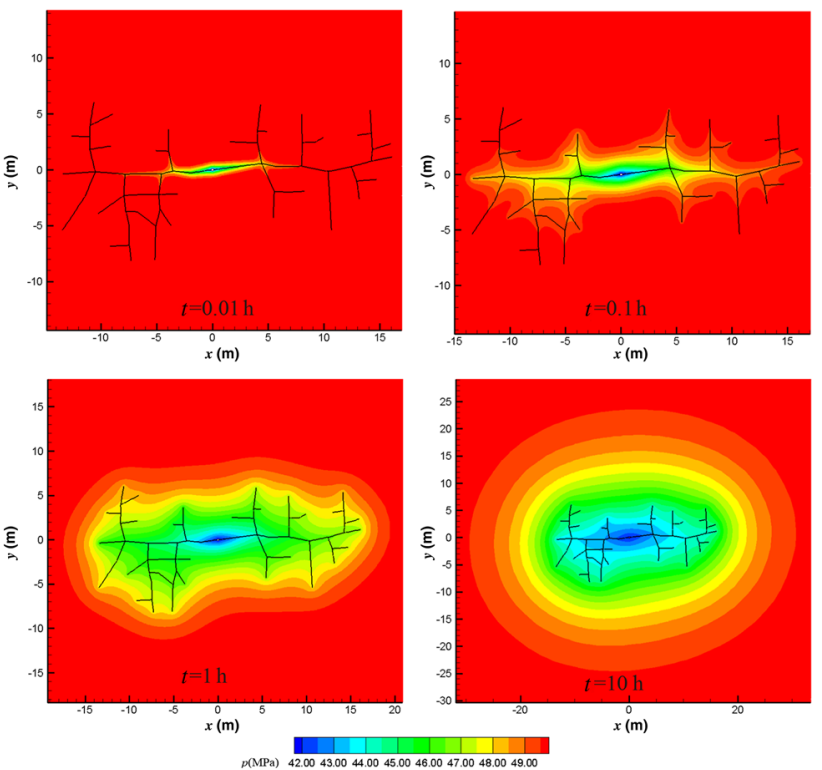

Fig. 16 (Color online) Pressure field of fractures with branches

\section{Concluding remarks}

A numerical method for a complex fracture well is developed based on the discrete-fracture model in which the matrix is discretized by triangles and fractures are discretized by 
line elements. The model is solved by the hybrid elements finite-element method. The finite-element method allows for an explicit and accurate representation of the fractures. This numerical method provides a powerful tool to study the single flow in media with complex fractures. The accuracy of the approach is demonstrated through several examples with various values of fracture aperture, fracture conductivity, and fracture amount. Results of the discrete-fracture model agree very well with the stripe-fracture model and the analytical solutions.

The discrete-fracture-fracture approach has several advantages. The preprocessing is simple and does not require gridding inside the fractures, which avoids small 2-D elements inside the fractures. It improves the conditioning of the discrete operator. The number of meshes in discrete-fracture model is $10 \%$ less than the stripe-fracture model, and computational efficiency increases by about $50 \%$ in the multiple fractures case. It is more powerful for the complex fractures problems, like the non-uniform aperture fractures and the fractures with branches.

All these results correspond to 2-D configuration. However, concepts of the discrete-fracture model can be extended to three dimensional (3-D), and it is under way.

Acknowledgments This work was supported by the National Natural Science Foundation of China (Grant 51404232), the National Science and Technology Major Project (Grant 2011ZX05038003), and the China Postdoctoral Science Foundation (Grant 2014M561074).

\section{References}

1. Gringarten, A.C., Ramey, J., Raghavan, R.: Unsteady-state pressure distributions created by a well with a single infinite-conductivity vertical fracture. J. Pet. Technol. 14, 347-360 (1972). doi:10.2118/ 4051-PA

2. Heber Cinco, L., F Samaniego, V., N. Dominguez, A.: Transient pressure behavior for a well with a finite-conductivity vertical fracture. Acta Mech. Sin. 18, 253-264 (1978). doi:10.2118/6014-PA

3. Weiyang, X., Xiaoping, L.: Research on fractured horizontal wells productivity and productivity influence in shale gas reservoir. Paper SPE 167674 presented at SPE/EAGE European Unconventional Resources Conference and Exhibition, Vienna, 25-27 Feb 2014. doi:10.2118/167674-MS

4. AI Rbeawi, S, Tiab, D.: Partially penetrating hydraulic fractures: pressure responses and flow dynamics. Paper SPE 16450 presented at SPE Production and Operations Symposium, Oklahoma City, Oklahoma, 23-26 Mar 2013. doi:10.2118/164500-MS

5. Aimene, Y.E., Nairn, J.A.: Modeling multiple hydraulic fractures interacting with natural fractures using the material point method. Paper SPE 167801 presented at SPE/EAGE European Unconventional Resources Conference and Exhibition, Vienna. 25-27 Feb 2014. doi:10.2118/167801-MS

6. Liu, Y.W., Ouyang, W.P., Zhao, P.H., et al.: Numerical well test for well with finite conductivity vertical fracture in coalbed. Appl. Math. Mech. Engl. Ed. 35, 729-740 (2014). doi:10.1007/ s10483-014-1825-6
7. Tan, X., Li, X.: Transient flow model and pressure dynamic features of tree-shaped fractal reservoirs. J. Hydrodyn. Ser. B. 26, 654-663 (2014). doi:10.1016/S1001-6058(14)60072-X

8. Zhou, R., Liu, Y.W., Zhou, F.X.: Numerical solutions for the transient flow in the homogenous closed circle reservoirs. Acta Mech. Sin. 19, 40-45 (2003). doi:10.1007/BF02487451

9. Ran, Q.Q., Gu, X.H., Li, S.L.: A coupled model for multiphase fluid flow and sedimentation deformation in oil reservoir and its numerical simulation. Acta Mech. Sin. 13, 264-272 (1997). doi:10. 1007/BF02487708

10. Zheng, H., Liu, F., Du, X.L.: Complementarity problem arising from static growth of multiple cracks and MLS-based numerical manifold method. Comput. Methods Appl. Mech. Eng. 295, 150171 (2015). doi:10.1016/j.cma.2015.07.001

11. Ouyang, W.: A study of unsteady and non-isothermal flow in porous coalbed media. [Ph.D. Thesis], Chinese Academy of Science, Beijing (2014)

12. Moinfar, A., Narr, W., Hui, M.-H., et al.: Comparison of discretefracture and dual-permeability models for multiphase flow in naturally fractured reservoirs. Paper SPE 142295 presented at SPE Reservoir Simulation Symposium, Texas, 21-23 Feb 2011. doi:10. 2118/142295-MS

13. Ma, K., Ren, G., Matten, K., et al.: Modeling techniques for foam flow in porous media. J. Pet. Technol. 20, 453-470 (2014). doi:10. 2118/169104-PA

14. Noorishad, J., Mehran, M.: An upstream finite element method for solution of transient transport equation in fractured porous media. Water Resour. 18, 588-596 (1982). doi:10.1029/ WR018i003p00588

15. Baca, R., Arneet, R., Langford, D.: Modeling fluid flow in fractured-porous rock masses by finite-element techniques. Int. J. Num. Methods Fluids. 4, 337-348 (1984). doi:10.1002/fld. 1650040404

16. Kim, J., Deo, M.D., Langford, D.: Finite element, discrete-fracture model for multiphase flow in porous media. AIChE J. 46, 1120 1130 (2000). doi:10.1002/aic.690460604

17. Karimi-Fard, M., Firoozabadi, A.: Numerical simulation of water injection in fractured media using the discrete-fracture model and the galerkin method. SPE Reserv. Eval. Eng. 6, 117-126 (2003). doi: $10.2118 / 83633-P A$

18. Karimi-Fard, M., Durlofsky, L.J., Aziz, K.: An efficient discrete fracture model applicable for general purpose reservoir simulators. J. Pet. Technol. 9, 227-236 (2004). doi:10.2118/88812-PA

19. Zhang, Y., Yuan, X.C., Yao, J., et al.: Discrete fracture numerical simulation methods for reservoirs. J. Daqing Pet. Inst. 34, 80-85 (2010)

20. Hui, M.-H., Mallison, B., Heidary-Fyrozjaee, M., et al.: The upscaling of discrete fracture models for faster, coarse-scale simulations of IOR and EOR processes for fractured reservoirs. Paper SPE 166075 presented at SPE Annual Technical Conference and Exhibition, New Orleans, Louisiana, 30 Sept-2 Oct 2013 doi:10.2118/ 166075-MS

21. Hazlett, R.D., Krishna Babu, D.: Discrete wellbore and fracture modeling for unconventional wells and unconventional reservoirs. Paper SPE 159379 presented at SPE Annual Technical Conference and Exhibition, San Antonio, Texas, 8-10 Oct 2012. doi:10.2118/ 159379-MS

22. Li, X.S., Demmel, J., Gilbert, J., et al.: http://crd-legacy.lbl.gov/ $\sim$ xiaoye/SuperLU/ 